\title{
Students' Perceptions of Clinical Education during a Global Pandemic
}

\author{
Pamela Pologruto $^{1}$ Jennifer Jewell ${ }^{1}$ Laura Cruz ${ }^{2}$ \\ 1 Physical Therapist Assistant Program, The Pennsylvania State \\ University, Pennsylvania, United States \\ 2 Schreyer Institute-Teaching Excellence, The Pennsylvania State \\ University, Pennsylvania, United States \\ Address for correspondence Pamela Pologruto, PT, DPT, Physical \\ Therapist Assistant Program, The Pennsylvania State University, Penn \\ State Fayette, 2201 University Drive, Lemont Furnace, PA 15456, \\ United States (e-mail: pjp17@psu.edu).
}

J Health Allied Sci ${ }^{\mathrm{NU}}$ 2022;12:150-154.

\begin{abstract}
Keywords

- clinical education

- COVID-19

- health-care literacy

- physical therapist assistant

Introduction Clinical education is an essential component of allied health programs. The coronavirus disease 2019 (COVID-19) global pandemic had significant impacts on clinical education. The central purpose of this research was to recognize student perceptions of the impact of COVID-19 on their face-to-face clinical experiences during the global pandemic.

Methods A Qualtrics survey was sent to 80 physical therapist assistant students whose clinical education was affected during the 2019 to 2020 academic year. Subjects were asked about the following: factors that influenced their decision to complete clinical rotations during the pandemic; perceptions of learning, safety, and stress under these conditions as well as perceptions of the effectiveness of clinical preparatory activities.

Results Twenty-six responses to the survey were received and analyzed using descriptive statistics and nonpaired $t$-tests calculated for each scaled survey item to compare groups. A majority (83.3\%) of the respondents reported graduation followed by professional experience (58.3\%) as extremely important factors of consideration for participation in clinical education. Personal safety (33.3\%) ranked the lowest of the extremely important factors. In preparing for the clinical experience, $75 \%$ reported that individual or small group meetings with the faculty member were extremely helpful. The majority of students (78.26\%) strongly agreed that they learned a great deal from the clinical experiences under global pandemic; however, $52.17 \%$ reported they found the clinical experiences to be stressful.

Conclusion During the global pandemic, students placed greater value on interpersonal, trusted relationships when seeking information. Even though students felt stressed during their clinical education, they perceived that learning did occur. As the impacts of COVID-19 remain, physical therapy programs can use this data to recognize areas that require increased support and preparation for students' clinical experiences to encourage an impactful and sustainable future in clinical education.
\end{abstract}

DOI https://doi.org/ $10.1055 / \mathrm{s}-0041-1736282$ ISSN 2582-4287.

\footnotetext{
(c) 2021. Nitte (Deemed to be University). All rights reserved. This is an open access article published by Thieme under the terms of the Creative Commons Attribution-NonDerivative-NonCommercial-License, permitting copying and reproduction so long as the original work is given appropriate credit. Contents may not be used for commercial purposes, or adapted, remixed, transformed or built upon. (https://creativecommons.org/ licenses/by-nc-nd/4.0/) Thieme Medical and Scientific Publishers Pvt. Ltd., A-12, 2nd Floor, Sector 2, Noida-201301 UP, India
} 


\section{Introduction}

Clinical education has long been considered an integral part of the educational process for students in allied health professions to master entry-level skills. The clinical experience typically serves to provide emerging health-care workers with the means to apply what they have learned in didactic courses to real-world settings and to immerse them in the kinds of workplace environment that they hope to occupy as professionals. Over the past few years, the practice of clinical education has been the subject of some debate, suggesting that some clinical experiences may be more effective at meeting desired learning outcomes than others. ${ }^{1,2}$ These conversations are ongoing, but one conclusion seems to be that academic programs may need to be more proactive in structuring and scaffolding the clinical experiences both for students and for those clinical sites that receive them.

The conditions fostered by the coronavirus disease 2019 (COVID-19) global pandemic have placed additional stress on the question of the value and best practices of clinical education. Many clinical sites canceled, postponed, and/or restricted activities due to COVID-19 restrictions. This significantly impacted allied health programs' ability to offer traditional clinical experiences for their students. In Spring 2020, the Commission on Accreditation for Physical Therapy Education reported the requirement of clinical education hours for physical therapist assistant (PTA) students included providing direct patient care and could not be fully meet with simulation. Fulfilling this requirement during the global pandemic required universities/colleges, faculty, and students to make decisions and adaptations to ensure students were able to fulfill requirements to graduate.

While research on the impact of COVID-19 and clinical education is only just starting to emerge, preliminary studies affirm the existence of significant deficits in clinical education. A 2020 study of 300 fourth- and fifth-year medical students, for example, indicated that over $68 \%$ of the respondents experienced a decrease in their clinical activities, largely due to the cancellation or reduction in opportunities across multiple sites. ${ }^{3}$ A second study of 440 fifth-year medical students also indicated a considerable reduction, with $75 \%$ of respondents reporting diminished activity. $^{4}$

The challenges to clinical education under COVID-19 conditions were not confined to the reduced availability of clinical sites (i.e., supply), but also in demand, as many students and programs had concerns about the safety of clinical work. Compton et al conducted an electronic survey of medical students less than 1 month into the COVID-19 pandemic with approximately one-third $(n=63)$ of the students expressing a preference to not return to the clinical education setting. ${ }^{5}$ An anonymous survey of 316 third and fourth-year medical students, residents, and fellows, too, reported that students and trainees felt anxious and vulnerable in the clinical environment and this negative affect increased if they were serving on the front lines. ${ }^{6}$ The students who preferred not to return to the clinical setting cited not only their safety but also increased risks to patients as the students felt insufficiently trained to navigate the challenges afforded by the pandemic. ${ }^{6}$

As students in the health professions made decisions regarding participating in clinical education, it is important to determine the students' knowledge base of COVID-19 and the resources being used to obtain this information. As the pandemic progressed and more valid resources became available regarding COVID-19, students could potentially avail themselves of a wide range of resources to make informed decisions regarding the implementation of preventive measures. An early study in a dental school indicated that media outlets provided by the World Health Organization (WHO), Centers for Disease Control and Prevention (CDC), and Ministry of Health (MOH) were the three most common sources of information used by students, but other sources varied widely. ${ }^{7}$

In addition to the safety and availability of reliable information, the timing of students' clinical experience may have contributed to their concerns about entering the clinical environment. Increased concerns that were expressed early in the pandemic may have lessened as more research became available. Loch et al conducted a survey of dental students in mid-March 2020 reporting that $90 \%$ of students perceived their health to be at risk while working in the dental teaching clinics. Sixty-two percent were concerned they could transmit the disease to their family members and/or flatmates. ${ }^{7}$ Another survey conducted approximately 2 months later (mid-May 2020) of 305 dental students reported that 55\% ( $n=168$ ) did not consider themselves prepared for the pandemic and $88 \%(n=269)$ reported having fear of transmitting the virus to family and friends. Some students (33.8\% [ $n=103])$ perceived COVID-19 to be very dangerous and $62.3 \%$ ( $n=109$ ) reported moderately dangerous. Eighty-two percent $(n=250)$ preferred to avoid working with COVID-19 suspected patients. ${ }^{8}$

Clinical education is an essential component of allied health education; however, completing them during a global pandemic may have changed the learning experience. Choi et al distributed a survey to final-year medical students who were asked whether assisting in hospitals during the COVID19 pandemic supplemented learning; 26.5\% $(n=116)$ strongly agreed and $44.5 \%(n=196)$ agreed. Students additionally were asked if they felt less prepared due to COVID-19 with $18.6 \%(n=82)$ strongly agreed and $40.7 \%(n=179)$ agreed. ${ }^{4}$ Loch et al's study reported that $53 \%$ of students considered their clinical performance to be negatively affected by the COVID-19 pandemic. Eighty-seven percent of the students reported increased stress during the pandemic. Students expressed stress over clinical cancellations and fulfilling requirements to graduate. ${ }^{7}$ Being overwhelmed with new COVID-19 regulations was also commonly reported. ${ }^{8}$ Another study of medical students found that $51 \%$ reported fear of being unprepared in the future and $59 \%$ reported a qualitative decline in education. ${ }^{3}$

Knowing students' perceptions of the clinical experience during a pandemic can help assist allied health programs with preparing students. The central purpose of this research is to recognize the allied health student perceptions of the 
impact of COVID-19 on their face-to-face clinical experiences during the pandemic. The conditions of the pandemic came about quite suddenly, so there are no available studies that consider the revision of clinical education in the context of the current age of crises involving PTA students. The study is intended to shed light on the factors that enable or inhibit the successful outcomes of clinical learning experiences under crisis conditions, with the intention of providing insight into strategies that could improve these outcomes in the future. While a vaccine for COVID-19 exists, it may not be the last health crisis we face, and it is unlikely that health-care settings will return to the same operating procedures used before the outbreak.

\section{Methods}

Penn State University is a multicampus, land-grant, public research university providing access to education throughout the Commonwealth of Pennsylvania. ${ }^{9}$ The institution is classified as a state-related university, meaning that it is not state-owned but maintains the character of public universities receiving state appropriations. ${ }^{9}$ There are 24 physical campuses across Penn State as well as a global online program. PTA programs are located on five campuses (Fayette, Shenango, Mont Alto, Hazleton, and DuBois) geographically dispersed across the state. All together there were 80 PTA students whose clinical education was affected during the 2019 to 2020 academic year. Nontraditional (defined as 25 years and older) students comprise roughly a third of students enrolled in PTA programs.

Penn State's PTA program is structured as a five-semester sequence that culminates in an associate degree. Following three semesters of didactic instruction, students complete a 3-week interim clinical experience followed by an additional semester of didactic coursework. Students then finish the program with two-terminal, 6-week clinical experiences. At the inception of the COVID-19 crisis in the spring semester of 2020 , students of all five programs had already completed their interim clinical experiences and had begun semester four of on-campus didactic instruction. On March 13, 2020, there was an immediate and abrupt switch to remote learning for all curricular content listed through the remainder of the spring semester. Students were scheduled to begin clinical experiences in early May; however, those were delayed as mechanisms were developed to ensure student safety within clinical settings by the university.

To investigate how the PTA students navigated this changing environment, the researchers developed an electronic survey disseminated through Qualtrics Survey Software (-Supplementary Appendix A, available in the online version). The final survey consisted of five demographic questions, twenty scaled items, and three open-ended questions. After receiving IRB approval, the survey was sent to all students enrolled in the PTA program at Penn State for the 2019 to 2020 academic year. In November of 2020 , the researchers sent a recruitment email to all active instructors of PTA clinical practicum. These instructors were asked to provide the recruitment email, including the survey link, to all students enrolled in a Clinical Practicum Course for either the Summer or Fall of 2020. Students consented to the survey through participation.

The researchers received 26 responses to the survey, constituting a $32.5 \%$ response rate. It should be noted that the response rate is approximate, as it is not known how many instructors forwarded the email request. All respondents were PTA majors, $84.6 \%(n=22)$ were females in the age range of 18 to 24 years old $(73.1 \%, n=19)$, a demographic profile that roughly commensurates with overall patterns within the program. The majority of the respondents completed their clinical experiences during Fall 2020 (58.3\%, $n=14)$ with $37.5 \%(n=9)$ completing clinical experiences in Summer 2020. Responses to the survey were analyzed using descriptive statistics, including mean, median, and standard deviation, where applicable. Further analysis was conducted to compare results between groups, with nonpaired $t$-tests calculated for each scaled survey item.

\section{Results}

The survey revealed results in four categories: personal safety, preparation, perception of learning, and stress.

\section{Personal Safety}

The survey asked respondents to rank the importance of a series of factors in deciding whether or not to participate in clinical rotations that required them to provide direct patient care under global pandemic conditions. Somewhat surprisingly, $83.3 \%(n=20)$ of the respondents reported graduation as an extremely important factor followed by professional experience $(58.3 \%, \quad n=14)$ closely followed by personal/family responsibilities $(52.2 \%, n=12)$. Convenience $(21.43 \%, n=3)$ and personal safety $(33.3 \%, n=8)$ ranked the lowest of extremely important factors. When analyzed between groups, finances played a more statistically significant role for students who chose to do their clinical rotation over the Summer versus the Fall. Additionally, nontraditional students placed statistically greater emphasis on finance, convenience, professional experience, and personal/family responsibilities than traditional students ( $\mathbf{- T a b l e ~} \mathbf{1}$ ).

The researchers assumed that students who postponed their clinical rotations to the Fall semester did so largely because of the risk of personal safety. During the Summer of 2020, COVID19 was a novel virus and clinical safety protocols were still being developed at many sites, a state of affairs that had largely been rectified by the start of the Fall semester. However, no statistically significant difference was found between students who completed clinical experiences in Summer 2020 versus Fall 2020 regarding safety as a factor of consideration. Overall, $60.87 \%(n=14)$ strongly agreed that they felt safe during their clinical rotation under global pandemic conditions with no students disagreeing with this statement.

\section{Preparation}

Respondents were asked to rank how helpful a list of activities were in preparing the student for a clinical experience during a global pandemic. Seventy-five percent $(n=18)$ reported that individual or small group meetings with 
Table 1 Statistically significant differences among timing of clinical and age of student

\begin{tabular}{|c|c|c|c|c|c|}
\hline$p$-Value & Survey question & $\begin{array}{l}\text { Summer } 2020 \\
\text { average }\end{array}$ & $\begin{array}{l}\text { Fall } 2020 \\
\text { average }\end{array}$ & $\begin{array}{l}\text { Traditional } \\
\text { student } \\
\text { average }\end{array}$ & $\begin{array}{l}\text { Nontraditional } \\
\text { student } \\
\text { average }\end{array}$ \\
\hline & $\begin{array}{l}\text { How important were each of the following factors } \\
\text { when you made your decision to participate in a } \\
\text { clinical rotation providing direct patient care under } \\
\text { global pandemic conditions? }\end{array}$ & & & & \\
\hline$p=0.15$ & - Finance & 2.77 & 1.93 & 2.53 & 1.43 \\
\hline$p=0.15$ & - Convenience & & & 2.94 & 1.86 \\
\hline$p=0.15$ & - Professional experience & & & 1.82 & 1.14 \\
\hline \multirow[t]{2}{*}{$p=0.15$} & - Personal/family responsibilities & & & 2.19 & 1.29 \\
\hline & $\begin{array}{l}\text { How helpful were each of the following activities in } \\
\text { preparing for clinical rotation under global pan- } \\
\text { demic conditions? }\end{array}$ & & & & \\
\hline$p=0.15$ & $\begin{array}{l}\text { - Communications from University senior } \\
\text { leadership }\end{array}$ & 4.44 & 3.14 & & \\
\hline$p=0.15$ & - Financial aid information & 3.14 & 2.86 & & \\
\hline$p=0.15$ & $\begin{array}{l}\text { I learned as much from my clinical experience under } \\
\text { global pandemic conditions than I would have under } \\
\text { normal circumstances }\end{array}$ & & & 2.06 & 1.29 \\
\hline$p=0.15$ & $\begin{array}{l}\text { I found my clinical experience under global pan- } \\
\text { demic conditions to be stressful }\end{array}$ & 3.22 & 2.07 & & \\
\hline
\end{tabular}

faculty members were extremely helpful to very helpful; this category was followed by independent reading $(70.8 \%$, $n=17)$ and consultation with family members $(66.7 \%$, $n=16)$. A statistical difference $(p=0.15)$ was found between the respondents who completed their clinical experience in Summer 2020 versus Fall 2020. Respondents who completed their clinical rotation in Fall 2020 reported that COVID-19 training from Penn State academic program was extremely to very helpful $(50 \%, n=7)$ versus Summer 2020 clinical experiences $(33 \%, n=3$ ) (-Table 1 ). Students completing clinical experiences during the Fall semester were provided with more time and access to additional trainings to aid in preparation. Similarly, the clinical sites also had more time to prepare and offer additional trainings for students in the Fall, with $57.2 \%(n=8)$ of respondents indicating that clinical site COVID-19 training was extremely helpful to very helpful compared to $33 \%(n=3)$.

\section{Perception of Learning}

The researchers also queried respondents about their perceptions of learning in clinical experiences under the circumstances of a global pandemic. The majority of students $(78.26 \%$, $n=18$ ) strongly agreed that they learned a great deal from the clinical experiences under global pandemic conditions and no respondents disagreed with the statement. A difference was noted in the respondents completing Summer 2020 clinical experience (66.67\% [ $n=6]$ strongly agreed, 22.22\% [ $n=2]$ somewhat agreed, $11.11 \%$ [ $n=1]$ neither agreed or disagreed) versus Fall 2020 clinical experience (85.71\% [ $n=12$ ] strongly agreed, $14.29 \%$ [ $n=2]$ somewhat agreed). However, when students were asked to compare their learning under pandemic conditions to learning under normal circumstances, $60.87 \%$
( $n=14)$ agreed that they learned as much as under normal conditions with $17.39 \%(n=4)$ somewhat agreeing and $21.74 \%$ $(n=5)$ somewhat disagreeing. No significant differences were found between the respondents participating in Fall 2020 versus Summer 2020 clinical experiences. Nontraditional students did show higher rates of agreement (than traditional students) with the statement that they learned as much under pandemic conditions as they would under normal conditions (-Table 1).

\section{Stress}

When respondents were asked if they found the clinical experiences to be stressful under global pandemic conditions, the responses significantly varied $(52.17 \%[n=12]$ strongly agreed to somewhat agreed, $26.09 \%$ [ $n=6]$ neither agreed or disagreed, and $21.47 \%$ somewhat disagreed to strongly disagreed $[n=5])$. Statistical difference $(p=0.15)$ was found between Summer 2020 clinical experiences with $22.22 \%(n=2)$ strongly agreed to somewhat agreed versus Fall 2020 with $71.43 \%(n=10)$. Although there are indications of increased stress when students were asked if they could turn back time, would they make the same decision to participate in these clinical experiences, $87 \%$ agreed or strongly agreed, with no students in disagreement.

\section{Discussion}

Traditionally, PTA students at Penn State complete their terminal clinical experiences in the Summer as the last requirement to fulfill to graduate. During Spring 2020, the COVID-19 global pandemic started that caused abrupt changes to the curriculum and clinical experiences. PTA 
students were given the option to complete their clinical experiences in Summer 2020 or postpone the clinical experiences until Fall 2020. Students were provided with the most current status, changes, and information related to the COVID-19 curriculum changes in different platforms including individual/small group meetings with PTA faculty along with information from Penn State administration and resources not associated with Penn State.

This study showed that proximity and trust played significant roles in how students exercised health-care literacy. Overall, they placed greater value on interpersonal, trusted relationships, especially with either faculty members with whom they had an ongoing relationship, or with family members. From their perspective, the most effective communication regarding their clinical experience came from individual or small group meetings with program faculty. PTA programs at Penn State are located on small campuses with a smaller cohort of students. This intimate atmosphere allows the opportunity for faculty to get to know individual students not just as learners, but as people with needs such as family, living, and financial situations. Students placed significantly less value on communications from the university (which enrolls close to 100,000 students) or from external news and information sources.

During Summer 2020, a higher percentage of respondents indicated that they felt unsafe during their clinical experiences, as compared to the Fall cohort. This difference is likely due to the increased availability of reliable information as well as the increased experience of the stakeholders involved. The initial transition period was characterized by a great deal of uncertainty, which lessened over time. That said, although the students felt safer in their Fall clinical placements, their perception of learning decreased. The conditions present in the clinical environment during the Summer of 2020 lead to significant and frequent changes in the health-care environment due to the changing protocols that fluctuated based on the availability of research. At this time, the health-care environment may have been less conducive to student learning due to the stressful and changing environment. Further, frequent health-care changes during this crisis may have limited staff time for student teaching and limited opportunities.

The majority of respondents that postponed their clinical experiences and graduation to Fall 2020 stated that they felt safe during their clinical rotation under global pandemic conditions. Fall 2020 respondents reported COVID-19 training from the university's academic program was more helpful as these students had more opportunities to prepare and participate in trainings versus the Summer 2020 students. A majority of Fall 2020 respondents reported that they learned a great deal from clinical experiences under global pandemic conditions. Decreasing uncertainty at this time gave the opportunity and time to provide students with more support.

When students decided to postpone their clinical experience to Fall 2020, the general assumption was that the student was concerned with their safety or had financial concerns. However, this study indicated that the main concerns were graduation and professional experience. Safety rated low for consideration to decide when to complete clinical experiences.

Even under the unprecedented conditions of COVID-19, this study suggests that our students were able to engage deeply in their clinical experiences. Potential stress over safety conditions appears to have been largely ameliorated by close communication with responsive faculty members and clinical site coordinators. This global pandemic taught student adaptability as the situation continued to change and evolve. This is a critical skill for health-care workers to have which presents the question, "How do we teach student resiliency in the absence of a crisis?"

\section{Note}

Penn State Institutional Review Board approved this study and deemed it as exempt.

\section{Authors' Contributions}

P.P., J.J., and L.C. have substantially contributed to the underlying research, data analysis, and drafting this manuscript.

Conflict of Interest

None declared.

\section{References}

1 Ironside PM, McNelis AM, Ebright P. Clinical education in nursing: rethinking learning in practice settings. Nurs Outlook 2014;62 (03):185-191

2 Kantar LD, Ezzeddine S, Rizk U. Rethinking clinical instruction through the zone of proximal development. Nurse Educ Today 2020;95:104595

3 Kapila V, Corthals S, Langhendries L, Kapila AK, Everaert K. The importance of medical student perspectives on the impact of COVID-19. Br J Surg 2020;107(10):e372-e373

4 Choi B, Jegatheeswaran L, Minocha A, Alhilani M, Nakhoul M, Mutengesa $\mathrm{E}$. The impact of the COVID-19 pandemic on final year medical students in the United Kingdom: a national survey. BMC Med Educ 2020;20(01):206

5 Compton S, Sarraf-Yazdi S, Rustandy F, Radha Krishna LK. Medical students' preference for returning to the clinical setting during the COVID-19 pandemic. Med Educ 2020;54(10):943-950

6 Gallagher TH, Schleyer AM. We signed up for this! - student and trainee responses to the COVID-19 pandemic. N Engl J Med 2020; 382(25):e96

7 Loch C, Kuan IBJ, Elsalem L, Schwass D, Brunton PA, Jum'ah A. COVID-19 and dental clinical practice: students and clinical staff perceptions of health risks and educational impact. J Dent Educ 2021;85(01):44-52

8 Alawia R, Riad A, Kateeb E. Risk perception and readiness of dental students to treat patients amid COVID-19: implication for dental education. Oral Dis 2020. Doi: 10.1111/odi.13593

9 Mission and Values: Penn State. Accessed April 15, 2021 at: https://www.psu.edu/this-is-penn-state/mission-and-values/ 